\title{
Perspective
}

PERSPECTIVE Actualité en histoire de l'art

1 | 2007

Antiquité/Moyen Âge

\section{Aux sources de la tradition de l'architecture en Occident : deux études de réception}

\section{Alain Schnapp}

\section{(2) OpenEdition}

1 Journals

Édition électronique

URL : http://journals.openedition.org/perspective/3729

DOI : 10.4000/perspective.3729

ISSN : 2269-7721

Éditeur

Institut national d'histoire de l'art

\section{Édition imprimée}

Date de publication : 31 mars 2007

Pagination : 54-58

ISSN : 1777-7852

\section{Référence électronique}

Alain Schnapp, "Aux sources de la tradition de l'architecture en Occident : deux études de réception », Perspective [En ligne], 1 | 2007, mis en ligne le 31 mars 2018, consulté le 01 octobre 2020. URL : http:// journals.openedition.org/perspective/3729; DOI : https://doi.org/10.4000/perspective.3729 


\title{
Aux sources de la tradition de l'architecture en Occident : deux études de réception
}

\author{
Alain Schnapp
}

\section{RÉFÉRENCE}

Lukas Clemens, «Tempore Romanorum constructa ». Zur Nutzung und Wahrnehmung antiker Überreste nördlich der Alpen während des Mittelalters, Stuttgart, Anton Hiersemann, 2003. 565 p., 32 fig. n. et b. ISBN : 3-7772-0301-7; 188,00 €.

Pierre Gros, Palladio e l'antico, Venise, Marsilio, 2006. 97 p., 69 fig. n. et b. ISBN :

$8831789090 ; 25 €$.

1 Deux livres qui viennent de paraître à trois ans d'intervalle contribuent à renouveler notre intelligence de la relation au passé, et particulièrement aux vestiges des architectures anciennes, des hommes du Moyen Âge et de la Renaissance. Ces deux études ont en commun une maîtrise exceptionnelle des sources tant textuelles qu'archéologiques. Leur méthode comme leur champ d'intérêt sont différents, mais ils constituent tous deux des ouvrages indispensables à qui souhaite comprendre le poids de la tradition et la relation des hommes au passé antique après la fin de l'Antiquité même. Alors que l'érudition a privilégié jusqu'à présent les sources écrites, ces deux essais offrent la particularité de renouveler complètement le sujet par une double approche de la tradition et des données archéologiques.

2 Le livre de Lukas Clemens, Tempore Romanorum constructa, porte le sous-titre «De l'utilisation et de la perception des vestiges antiques au Nord des Alpes durant le Moyen Âge ». L'auteur a réalisé un véritable travail de bénédictin en collectant une impressionnante quantité de témoignages concernant les édifices antiques et en les rapprochant chaque fois qu'il le pouvait des dossiers archéologiques les plus fournis. L'ouvrage s'ouvre par une présentation historiographique et méthodologique et se 
divise ensuite en deux grandes parties : l'une consacrée à l'histoire même d'un certain nombre de sites et de monuments antiques au Moyen Âge, l'autre à leur perception. L'histoire monumentale se transforme ainsi en une histoire de la réception. L'auteur maitrise une documentation encyclopédique, il ajoute à son savoir-faire une réflexion historiographique qui donne à ce livre une place de choix aux côtés du fameux essai de Jean Adhémar ${ }^{1}$ qui était jusqu'ici la référence, malheureusement limitée à l'art français, et aux trois volumes de Salvatore Settis qui couvraient pour une période plus large la péninsule italienne ${ }^{2}$.

3 L'auteur allie à son savoir philologique une vaste culture archéologique. Il commence par des études de cas : Metz, Besançon, Reims, Cologne, Mayence, Trêves font l'objet d'une présentation systématique. Ces villes qui ont joué un rôle important à la fin de l'empire romain, se prêtent particulièrement à ce type d'approche, et sont ensuite intégrées dans une réflexion plus générale. L'auteur insiste sur le paysage des ruines et la culture des ruines qui dominent l'évolution historique de ces anciennes civitates. La prégnance de l'urbanisme romain comme la conscience avérée des limites de la ville au Bas Empire exercent partout des conséquences structurales tant sur le projet urbain que sur les pratiques architecturales. L'auteur poursuit sa réflexion en donnant une typologie fonctionnelle du devenir des principaux monuments des villes romaines: amphithéâtres, thermes, fora et capitolia, installations hydrauliques. Le fameux texte classique de Sigebert de Gembloux en 1070 (la continuation de la Chronique d'Eusèbe de Césarée) est un exemple littéraire bien attesté, dont L. Clemens montre qu'il était loin d'être isolé, tout autant que les célèbres Laudes d'Alexander Neckam. L'intérêt des clercs du Moyen Âge pour les thermes est remarquable, et s'affirme dans la réutilisation de ces ensembles pour y bâtir des églises ou des palais princiers. Moins connue est l'évolution des fora dont Clemens démontre qu'ils sont souvent transformés en fortifications mais qu'ils peuvent continuer à servir, souvent dans le sud de la France, d'entrepôts à vocation commerciale, voire de lieux de réunion. Le sort des installations hydrauliques est, lui, beaucoup moins glorieux, faute d'entretien, sauf de rares exceptions.

4 L'un des chapitres les plus originaux du livre est consacré aux édifices en zones non urbaines. Pour ceux-ci, la documentation écrite fait largement défaut, mais les sources archéologiques montrent bien la pérennité des édifices romains dans les campagnes et leur utilisation comme marqueurs territoriaux, ainsi que comme limites de zones d'influences économiques ou politiques. L'étude des camps militaires et des sanctuaires ruraux contribue largement à mieux définir la variété des remplois et des usages de l'architecture romaine dans les campagnes. Un chapitre est ensuite dédié aux usages des ruines antiques: carrières, four à chaux, réutilisation des tuiles et des briques, extraction de matériaux divers et leur réemploi.

5 Sur cet inventaire solide, L. Clemens bâtit ensuite une ambitieuse histoire de la réception des ruines antiques qui prend en compte tant les sources ecclésiastiques que les traditions historiques. L'hagiographie apporte, si l'on peut dire, sa pierre à la construction en nous faisant découvrir combien la fondation de monastères dans des ruines antiques est une pratique courante. L'auteur reconstruit un paysage intellectuel négligé, celui de la connaissance des sites antiques par les clercs du Moyen Âge, et il décline son approche en reprenant certains thèmes de l'historiographie et de la légende de Jules César pour s'intéresser aux pratiques antiquaires médiévales. La conscience aiguë du passé romain dans les Flandres et l'intégration de l'histoire 
monumentale antique dans la restructuration de l'espace constituent un idéal type qui dément la tradition "pirennienne » de la rupture ${ }^{3}$. Le concept même, rendu classique par les travaux de Wilhelm Heckscher ${ }^{4}$, est remis en cause par l'analyse de certaines émissions monétaires des $\mathrm{XI}^{\mathrm{e}}$ et $\mathrm{XII}^{\mathrm{e}}$ siècles qui reprennent la titulature romaine, ou par la sigillographie (p. 376-380). Les exemples de découvertes fortuites d'antiquités, voire les excavations organisées dans le but d'en tirer un profit économique comme à Cologne, sont autant de démentis d'une profonde rupture (p. 391-393). Ces réflexions originales sont complétées par un intéressant dossier dédié à l'interprétation d'inscriptions antiques par les clercs médiévaux. La coupure entre tradition antique et curiosité médiévale apparaît ainsi beaucoup plus tardive qu'admise de façon traditionnelle, et peut être assignée au XIII siècle comme l'avait déjà suggéré Adhémar.

6 Le livre de L. Clemens est une somme qui repose sur des informations de première main. Il renouvelle complètement un sujet relativement négligé par l'érudition et il mêle avec bonheur histoire des sites, histoire de la réception des antiquités et histoire intellectuelle.

7 La découverte, la réception et l'utilisation de la tradition de l'antiquité classique ne sont pas, on l'a vu, la conséquence d'un seul mouvement de curiosité. Le rapport à l'antique est une donnée immédiate de la conscience de soi dans des paysages dominés par les monuments anciens comme la Rome du XIve siècle. Pour les hommes de la Renaissance, le passé est un territoire qu'il faut explorer, un vaste champ de ruines que les esprits éclairés peuvent transformer en chambre des merveilles au sens matériel du terme quand les antiquités sont rassemblées en collection, ou au sens immatériel quand elles sont réunies en un vaste corpus de savoir. De ce point de vue, les architectes sont à mi-chemin de l'un et de l'autre. Sans savoir, donc sans connaissance de la tradition architecturale antique, ils ne peuvent comprendre les ruines. Inversement ils ne peuvent interpréter les rares textes à leur disposition sans l'épreuve du réel, sans en passer par la construction de bâtiments inspirés par les exemples de l'Antiquité dont les traces, à Rome et dans la campagne romaine, étaient si impressionnantes.

8 L'intérêt du livre de Pierre Gros admirablement édité par le Centro Internazionale di Studi di Architettura Andrea Palladio est de nous faire pénétrer dans l'intimité d'un des architectes les plus créatifs de son temps dont l'œuvre est une constante redécouverte de l'architecture de l'Antiquité.

9 L'auteur entreprend une étude d'ensemble de la doctrine d'interprétation de Palladio et des hommes de son temps. Son livre est nourri de la connaissance des traités et relevés d'Alberti, de Sangallo et de Daniele Barbaro, mais il s'appuie directement sur l'œuvre de Vitruve et sur une expérience inégalée de l'architecture romaine et des techniques architecturales anciennes et modernes. Pour entrer dans la mécanique complexe de l'histoire de l'architecture moderne au moment même où elle s'émancipe de la tradition médiévale, il faut pouvoir tenir les deux bouts de la corde. Tout le mérite de P. Gros est d'allier la culture du philologue, l'œil de l'archéologue et l'expérience de l'architecture. Cela transparaît à chaque page de son travail, écrit dans un style efficace, fort bien traduit par Maria Beltramini.

D'emblée l'auteur expose un projet qui est en quelque sorte un travail de doctrine sur le vocabulaire, la méthode et les techniques de travail de Palladio. L'ouvrage est divisé en quatre chapitres; le premier, dédié au stylobate et aux fameux scamilli impares (" petites banquettes de hauteurs inégales ", selon les propres termes de P. Gros dans sa traduction de Vitruve), s'attaque à la question de la compréhension par Palladio du 
système de correction optique de l'assise des temples. Le second s'attache à définir la relation ambiguë des architectes de la Renaissance avec les temples des anciens, le troisième est consacré à la domus romaine et le dernier aux théâtres, tant dans l'œuvre de Vitruve que dans la pratique de relevé et d'interprétation.

11 Sous sa forme concrète et factuelle, le livre de P. Gros est en fait un traité de psychologie historique qui, pour chacun des types de monuments considérés, s'emploie à faire le point des outils à la disposition de Palladio et de ses contemporains. Il reconstitue leur champ d'interprétation et décrit les stratégies d'observation (qui incluent la lecture de Vitruve) et leurs conséquences sur les projets de construction.

12 L'interprétation des vestiges antiques par les architectes de la Renaissance relève d'abord d'une conscience de la beauté, de la dignitas et de la majestas des œuvres majeures de l'architecture antique. La fascination des architectes est liée en premier lieu aux techniques de relevé, à la possibilité de s'approprier par des mesures et des dessins un art antique qui est à la fois objet d'admiration et d'inquiétude. Car dans la présence matérielle des monuments, les clercs distinguent, sans toujours vouloir se l'avouer, la dimension païenne d'un art qu'il faut pourtant rapatrier dans le giron de la doctrine chrétienne. La supériorité de l'architecte sur le philologue ou l'antiquaire est de pouvoir organiser une confrontation en quelque sorte matérielle avec l'œuvre. Il ne doit pas seulement s'assurer comme l'antiquaire de l'intelligibilité de son relevé, il lui faut éprouver la réalité des techniques ou des dispositifs qu'il observe. D'où l'attitude complexe des architectes et de leurs donneurs d'ordre face aux « ruines de la Rome impériale considérées selon les cas comme des modèles qu'il est bon d'étudier, de mesurer et de reproduire et en même temps, d'espace à conquérir qu'il est parfaitement légitime de saccager » (p. 25).

13 Cette ambivalence est bien sûr liée à une forme nouvelle de rapport à la tradition. Pour les hommes du Moyen Âge, le passé constituait un énorme bloc de monuments et d'institutions dont seules certaines parties étaient intelligibles. L'effort des humanistes est de s'approprier le passé comme une matière première, tant pour l'édification concrète de la nouvelle Rome que pour la fondation intellectuelle d'une culture nouvelle. Les architectes sont au cœur même de ce processus de cognition, d'appropriation et bien sûr de destruction, et ce livre nous fait comprendre pourquoi.

La question des ordres, comme le rappelle P. Gros, est l'une des plus captivantes. Vitruve n'utilise pas le terme genus au sens d'ordre architectural tel qu'il apparait à la Renaissance, il n'applique cette notion qu'à l'architecture dorique et ionique, entendue comme l'expression de principes masculins et féminins, complémentaires et antagonistes. Le toscan n'est pour lui qu'une dispositio qui ne peut être mise sur le même plan que le genus dorique ou ionique (p. 26). Comme le souligne P. Gros, les « cinq ordres" (toscan, dorique, ionique, corinthien et composite) au sens canonique du terme sont une invention spécifique des architectes de la Renaissance. Palladio reprend et théorise cette clef d'interprétation de l'architecture des anciens mais en y ajoutant le concept de module qui permet d'unifier les proportions (et donc les relevés) des monuments en partant du diamètre inférieur de la colonne. Cette volonté d'élaborer un système descriptif unifié de l'ensemble des composantes de l'architecture est évidente dans le travail de Palladio et dans l'utilisation des relevés (qu'ils soient les siens propres ou ceux de ses contemporains). P. Gros détaille avec précision le système de rendu et les hypothèses géométriques qui sous-tendent la description des ordres dorique, ionique et corinthien. L'essentiel du chapitre est cependant consacré à la résolution de 
la difficile notion des scamilli impares, qui servent à redresser la perspective de la base des colonnes sur le stylobate. Après une analyse serrée du problème, l'auteur note que l'idée palladienne d'aller chercher les scamilli impares sur le podium du temple, immédiatement au contact des colonnes, «constitue, face aux interprétations courantes de l'époque, une petite révolution copernicienne » (p. 35).

Pour maîtriser l'héritage de l'architecture antique, Palladio devait disposer d'une méthode d'observation dont P. Gros reconstitue magistralement les étapes : vocabulaire de l'architecture, système d'interprétation du monument, concept d'ordre et recours tant aux relevés qu'aux expériences de construction. Il s'agit bien d'un système expérimental prébaconien dont la visée apparaît dans les trois chapitres successifs.

Le temple tout d'abord, en tant que manifestation la plus haute de l'art des anciens, est l'un des objets privilégiés de l'attention des architectes: à la fois du fait de la conservation parfois exceptionnelle de ces bâtiments à Rome et dans le reste de l'Italie, mais aussi parce que la commande architecturale de l'époque est liée au mouvement de reconquête tridentine. Bâtir des églises est l'un des outils de l'affirmation d'un catholicisme qui affronte la Réforme, fort de sa maîtrise de la capitale de l'Antiquité. Là encore, comme en matière d'ordre, il est fondamental de comprendre que le temple antique, contrairement aux présupposés des hommes de la Renaissance, n'est pas l'église chrétienne. L'intérêt, la passion même déployée par Palladio et ses contemporains dans l'étude des temples ne peuvent cependant pas s'entendre sans leur volonté de rabattre l'un sur l'autre. P. Gros souligne une fois de plus l'influence de Daniele Barbaro et de son cercle humaniste dans la réception par Palladio de la tradition écrite, mais il insiste aussi sur le rôle des fouilles. Palladio se réfère en effet précisément à la fouille sur le Forum du temple de Jupiter (aujourd'hui considéré comme le temple de Sérapis). Là où Sangallo voyait une domus, il est capable de reconnaître un temple et de reconstituer l'ordre interne de l'édifice sur la base des chapiteaux ioniques découverts. L'identification de la basilique de Maxence avec un temple de la paix, malgré l'erreur même d'interprétation due à Palladio, s'avère un élément de premier ordre pour reconstituer sa capacité d'observation et son intuition en matière technique.

Mais l'acuité palladienne va encore plus loin : il note au passage que le temple de la paix ne peut pas être attribué à l'époque de Vespasien et propose une datation plus tardive, à "un tempo che le cose de l'architettura non si intendevano cosi bene come al tempo di Vespasiano " [une époque où l'architecture n'était pas aussi bien entendue qu'au temps de Vespasien, p. 47]. Palladio s'emploie à construire un système descriptif qui établit des genres et des types de monuments, mais il s'approche plus précisément encore d'une technique archéologique, en ayant l'intuition d'une typologie chronologique.

Cette idée d'une sorte de stratigraphie conceptuelle des diverses parties du temple, de ce que nous appelons aujourd'hui une archéologie du bâti, s'exprime de façon éclatante dans son analyse exemplaire du « Tempietto » de Bramante à San Pietro in Montorio. La critique de Palladio érige cette construction en une sorte de commentaire more romano qui permet à travers une œuvre moderne de dégager les traits saillants de l'héritage classique. L'art de Bramante " uomo eccelentissimo e osservatore degli edifici antichi » [très remarquable observateur des édifices antiques] devient l'idéal type d'un nouveau modèle d'architecture plus antique dans sa conception que les œuvres de l'Antiquité elle-même. Bramante réussit alors aux yeux de ses contemporains « la christianisation de la sémantique vitruvienne ». 

dialogue contrasté de la culture occidentale avec le passé gréco-romain. Entre révérence et vandalisme, les attitudes des clercs et des architectes sont instables et parfois pour nous ambiguës, mais elles témoignent de la constance de la tradition et de la transmission d'un savoir qui passe par des voies souvent détournées. L'exploration du passé est plus riche d'arrangements, d'ajustements et de bricolages divers que de coupures épistémologiques ou de renversements historiographiques. La continuité l'emporte sur la rupture si on veut bien admettre que chaque génération a dû négocier le difficile équilibre de la mémoire et de l'oubli. On ne peut que souhaiter que ces deux livres deviennent, dans un jour pas trop éloigné, accessibles au public en langue française. 


\section{NOTES}

1. Jean Adhémar, Influences antiques dans l'art du Moyen Âge français (Londres, 1939) Paris, 1996.

2. Salvatore Settis éd., Memoria dell'antico nell'arte italiana, 3 vol., Turin, 1984-1986.

3. Henri Pirenne, dans son Mahomet et Charlemagne (1937), avait cherché à montrer la nouvelle identité du bassin méditerranéen partagé entre l'empire carolingien et le monde musulman mais en rupture profonde avec la civilisation antique.

4. Wilhelm Sebastian Heckscher : Die Romruinen: Die gei- stigen Voraussetzungen ihrer Wertung im Mittelalter und in der Renaissance, Wurzbourg, 1936.

INDEX

Keywords : remains, architecture, archaelogy, historiography, ruins

Mots-clés : vestige, architecture, archéologie, historiographie, ruines

Index chronologique : MOYEN ÂGE, RENAISSANCE

\section{AUTEURS}

ALAIN SCHNAPP

INHA 